imaging to the time domain. Imaging of very fast temporal events is a rapidly evolving field with applications as varied as imaging subpicosecond dynamics to imaging behind walls $^{6-8}$. Temporal ghost imaging has the potential for stimulating new ideas and opportunities for telecommunications and beyond.
Daniele Faccio is at the School of Engineering and Physical Sciences, Heriot-Watt University, Edinburgh EH14 4AS, UK.

e-mail:d.faccio@hw.ac.uk
2. Valencia, A., Scarcello, G., D’Angelo, M. \& Shih, Y. Phys. Rev. Lett. 94, 063601 (2005)

3. Ferri, F. et al. Phys. Rev. Lett. 94, 183602 (2005).

4. Ryczkowski, P., Barbier, M., Friberg, A. T., Dudley, J. M. \& Genty, G. Nature Photon. 10, 167-170 (2016)

5. Aspden, R. S. et al. Optica 2, 1049-1052 (2015).

6. Nakagawa, K. et al. Nature Photon. 8, 695-700 (2014).

7. Velten, A. et al. Nature Commun. 3, 745 (2012).

References

1. Pittman, T. B., Shih, Y. H., Strekalov, D. V. \& Sergienko, A. V. Phys. Rev. A 52, R3429(R) (1995).
8. Gariepy, G., Tonolini, F., Henderson, R., Leach, J. \& Faccio, D. Nature Photon. 10, 23-26 (2016).

\title{
IMAGING
}

\section{Super-resolution fight club}

A 2016 competition to find the optimum software for 3D single-molecule localization microscopy will help practitioners choose the best tool for the job and spur further developments in the field.

\section{Seamus Holden and Daniel Sage}

F or the popular super-resolution imaging technique of single-molecule localization microscopy ${ }^{1,2}$, accurately localizing the position of single fluorescent molecules is critical for success. In conventional biological imaging, the point spread functions of the fluorescent emission from each molecule are limited by diffraction and overlap with each other causing a loss of spatial resolution. The trick with localization microscopy is to start with all molecules in a dark, non-fluorescent state, and then stochastically activate only very small subsets (Fig. 1). If the emitting molecules of the subset are sufficiently isolated it is then possible to locate their position, which corresponds to the centre of the point spread function, with sub-diffraction-limited accuracy. Repeat this process over and over, activating and finding the positions of different subsets of

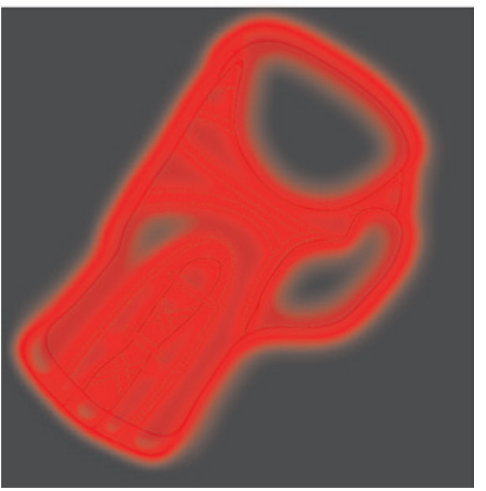

molecules until all the molecules have been localized, plot all those positions, and there you have it, a super-resolved image.

Since localization is so central to the success of the technique, it is unsurprising that developments in localization algorithms have played a key role in improving its performance, especially in speeding up the technique. By reducing the separation required between bright molecules to accurately find their centres ${ }^{3,4}$, so-called high-density localization algorithms have recently led to video-rate localization microscopy ${ }^{5}$. The speed of localization algorithms has also increased dramatically, with run times per image having dropped from hours to seconds ${ }^{6,7}$.

The Single Molecule Localization Microscopy Challenge was first run in 2013 and was deliberately limited to 2D datasets. It was designed to allow researchers in the field with a means to test and rigorously compare different localization software on biologically inspired simulated structures. Its goals were and still are (1) to help the non-expert user navigate the algorithmic maze by identifying the best tools for the job and (2) to identify common algorithmic limitations to spur further developments in the field. More than 30 research groups participated in the 2013 challenge and the results were published in Nature Methods in 2015.

One of the really interesting problems for the challenge, and indeed the field, is to establish valid ways of assessing the quality of super-resolution images and algorithms. Most localization microscopy algorithms do not actually output images; they output lists of localized molecule positions from which a b

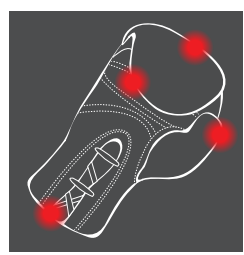

d

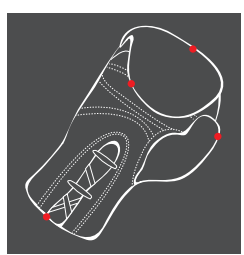

c

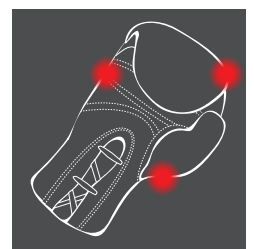

e



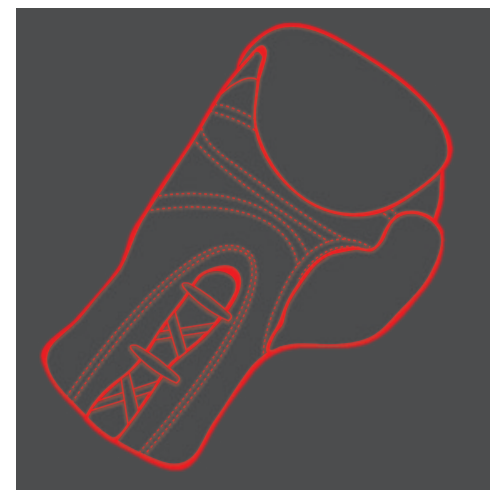

Figure 1 | The principle of super-resolution single-molecule localization microscopy. $\mathbf{a}$, Conventional image of closely spaced fluorescent molecules that form the shape of a boxing glove. The resolution of the image is limited by diffraction. $\mathbf{b}-\mathbf{e}$, By sequentially switching on and imaging different subsets of molecules (b,c), the location of each molecule, corresponding to the centre of the emitted fluorescence point spread function, can be precisely determined (d,e). $\mathbf{f}, \mathrm{A}$ superresolved image is created by plotting the positions of all localized molecules. 
final super-resolved image is reconstructed. The completely synthetic nature of the final image makes traditional analysis metrics such as the modulation transfer function ineffective. In fact, metrics for quantities as basic as spatial resolution have only recently been defined for localization microscopy ${ }^{9}$.

We are currently organizing the second edition of the challenge and the 2016 version will focus on 3D localization microscopy. While nanoscale-resolution $3 \mathrm{D}$ imaging is a powerful tool for studying protein ultrastructure in vivo, it also places significant extra demands on the image analysis.

3D localization microscopy works in a similar way to its $2 \mathrm{D}$ counterpart, but with the additional demand of encoding the axial position of a molecule in the shape of the point spread function. This extra requirement to estimate point spread function shape makes designing effective 3D algorithms much more challenging, particularly for low signal-to-noise data. Because of this, and probably because commercial 3D localization microscopy systems were less common until recently, 3D software has seen less development and characterization than its $2 \mathrm{D}$ cousin. We hope to remedy this by robust testing of existing algorithms, and by creating a series of openly available simulated datasets to support future development. We are particularly keen to see how different 3D software performs away from optimal focus, since this is crucial to the effective depth of field of the technique.

At the same time we will continue the previous $2 \mathrm{D}$ challenge, updating it with new datasets to reflect the evolving demands of the field. It will be interesting to see how new challengers fare against the previous crop.

We hope that the provision of openly available experimentally realistic test datasets will also encourage participation of the broader image processing community. Advances have previously come from other disciplines as diverse as astronomy and compressed sensing. Who knows where the next one will come from?

The 2016 Single Molecule Localization Microscopy Challenge will run from April to June, with the results to be presented at the 6 th Single Molecule Localization Microscopy Symposium, which is on 28-30 August at the EPFL, Lausanne, Switzerland (http://smlms.epfl.ch). Further details and registration information can be found on the challenge website (http://bigwww.epfl.ch/ $\mathrm{smlm} /$ challenge2016). Broad participation is warmly encouraged.

Seamus Holden is at the Centre for Bacterial Cell Biology, Institute for Cell and Molecular Biosciences, Newcastle University, Newcastle Upon Tyne NE2 4AX, UK. Daniel Sage is in the Biomedical Imaging Group, School of Engineering, Ecole Polytechnique Fédérale de Lausanne, CH-1015 Lausanne, Switzerland.

e-mail:seamus.holden@ncl.ac.uk; daniel.sage@epfl.ch

References

1. Betzig, E. Science 313, 1642-1645 (2006).

2. Rust, M. J., Bates, M. \& Zhuang, X. Nature Methods 3, 793-795 (2006)

3. Holden, S. J., Uphoff, S. \& Kapanidis, A. N. Nature Methods 8, 279-280 (2011).

4. Huang, F., Schwartz, S. L., Byars, J. M. \& Lidke, K. A. Biomed. Opt. Express 2, 1377-1393 (2011)

5. Huang, F. et al. Nature Methods 10, 653-658 (2013).

6. Wolter, S. et al. Nature Methods 9, 1040-1041 (2012).

7. Smith, C. S., Joseph, N., Rieger, B. \& Lidke, K. A. Nature Methods 7, 373-375 (2010)

8. Sage, D. et al. Nature Methods 12, 717-724 (2015)

9. Nieuwenhuizen, R. P. J. et al. Nature Methods 10, 557-562 (2013).

\section{PLASMONICS}

\section{Tunable spectrometer}

A miniature mid-infrared spectrometer that combines the advantages of actuated microelectromechanical systems (MEMS) with the sensitivity of plasmonics has been built by scientists from Boston University (ACS Photon. 3, 14-19; 2016). The device, a few hundred micrometres long and wide, consists of a gold film featuring a subwavelength hole array that is suspended above a gold reflector by an actuated polysilicon frame. Electrostatic actuation of the frame changes the gap between the gold film and the reflector, thus modifying the optical path length and the spectral response of the Fabry-Perot interferometer that they form.

"The additional mechanical degree of freedom provided by the MEMS will enable dynamic tuning of the spectral response of the system, making this device a powerful tool for differential measurements and spectrometry," commented Thomas Stark from Boston University.

In recent years, subwavelength-sized holes in metal films have proved to be useful in various sensing applications due to their ability to greatly enhance the

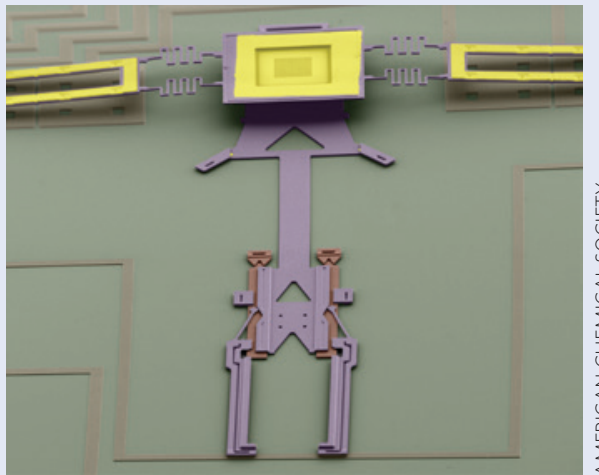

a suspended gold film, in which an array of rectangular holes was fabricated using focused ion beam milling. The polysilicon frame was suspended $21.67 \mu \mathrm{m}$ above the gold reflector. The size of the rectangular holes and the thickness of the gold film were $1,200 \mathrm{~nm} \times 500 \mathrm{~nm}$ and $100 \mathrm{~nm}$, respectively. The team says that the suspended design has two advantages. First, surrounding the nanoscale holes with a homogeneous dielectric background provides higher quality plasmonic resonances. Second, the use of the polysilicon frame prevents the

strength of the local electromagnetic field via the excitation of surface plasmons. This plasmonic enhancement can dramatically boost the sensitivity of absorption and Raman spectroscopy, allowing the techniques to reach new regimes of operation. However, the broad spectral width of a plasmon resonance means that it can be difficult to distinguish between closely spaced spectral absorption features.

In the Boston team's MEMS device (pictured), a polysilicon frame supported suspended gold film from being strained during the actuation of the MEMS device.

By tuning the distance between the gold film and the reflector from $1.7 \mu \mathrm{m}$ to $21.67 \mu \mathrm{m}$, the spectral response of the MEMS device was modulated. Combining this modulation with phase-sensitive detection methods makes it possible to enhance the device's signal-to-noise ratio.

NORIAKI HORIUCHI 